Absolute interferometric characterization of an x-ray mirror surface profile

This content has been downloaded from IOPscience. Please scroll down to see the full text. 2016 Metrologia 531

(http://iopscience.iop.org/0026-1394/53/1/1)

View the table of contents for this issue, or go to the journal homepage for more

Download details:

IP Address: 131.169.205.81

This content was downloaded on 14/12/2015 at 15:30

Please note that terms and conditions apply. 


\title{
Absolute interferometric characterization of an x-ray mirror surface profile
}

\author{
Maurizio Vannoni and Idoia Freijo Martìn \\ European XFEL GmbH, Albert-Einstein-Ring 19, 22761 Hamburg, Germany \\ E-mail: maurizio.vannoni@xfel.eu
}

Received 19 August 2015, revised 13 October 2015

Accepted for publication 6 November 2015

Published 15 December 2015

\begin{abstract}
An approach to achieve absolute planarity characterization of an x-ray mirror through an interferometric method is presented. With three measurements and two nominally flat auxiliary mirrors, the radius of curvature and the slope profile of a nominally flat x-ray mirror are retrieved. The height profile is then calculated through integration of the slope profile and merging this information with the radius of curvature knowledge. The method is explained in detail and a measurement example is provided. A comparison with a state-of-the-art deflectometric method is shown, with an agreement level of about $0.14 \mathrm{~nm} \mathrm{rms}$.
\end{abstract}

Keywords: interferometry, x-ray optics, flatness

(Some figures may appear in colour only in the online journal)

\section{Introduction}

The importance of having a reliable and very precise characterization of $\mathrm{x}$-ray mirrors is quite evident in synchrotron technique, where every small departure from a perfect surface is affecting the propagation of the beam and the quality of the focus. In particular for free electron lasers, the intrinsic spatial and temporal coherence of the beam produces an amplification of the distortions, with coherent effects rising up during the beam propagation. The resulting intensity patterns and focus shape modifications can put a strong limitation on the quality of the beam and therefore of the experiments. There are several projects of new x-ray facilities around the world, with always more challenging specifications, higher intensities and faster repetition rates, and the optics requirements are going in the same direction. One of the more common setups is to use a couple of offset mirrors to separate the $\mathrm{x}$-ray beam from spontaneous radiation, and at least an additional mirror to distribute the beam among the experimental stations. Because of the very low wavelength, x-ray mirrors are reflected in grazing incidence, with an angle value in the order of milliradians. That's also the main reason why they are designed to be long and narrow: being the incidence angle so close to $90^{\circ}$ in respect to the surface normal, the projected footprint needs a long mirror to avoid masking and to maintain a brilliant reflected beam. A secondary effect of such a setup is that every figure error of the mirror is desensitized by the shallow angle, and this effect partially compensates the higher sensitivity to the surface defects due to the very short wavelength. Despite that, the requirements for the mirrors are remaining tight and challenging, on the order of magnitude of few nanometers or few tenths of nanoradians peak-to-valley.

The material, which these mirrors are made of, is glass or silicon; the latter is usually preferred for its excellent mechanical and thermal properties. The surface is preliminarily polished with a classical mechanical polishing, then accurately measured, and finally polished with a deterministic polishing process, as ion-beam figuring [1, 2], atmospheric plasma-jet surface processing [3], or elastic-emission-machining [4]. The availability and potential high accuracy of these advanced polishing methods has reinforced the connection between manufacturing and surface metrology, and an accurate metrology of the mirrors has become the key factor to have the deterministic polishing fully effective. The common way to provide such metrology is Fizeau interferometry, even if new measuring approaches based on laser deflectometry are also possible [5] in particular for X-ray optics [6].

Fizeau interferometry is a relative technique: it is always a comparison between a test surface and a reference one. If the quality required for the test surface is close to or even better than that of the reference surface, an absolute method has to be used. 
1a)

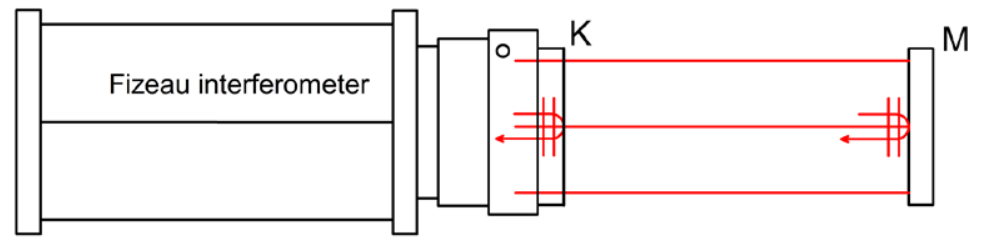

1b)

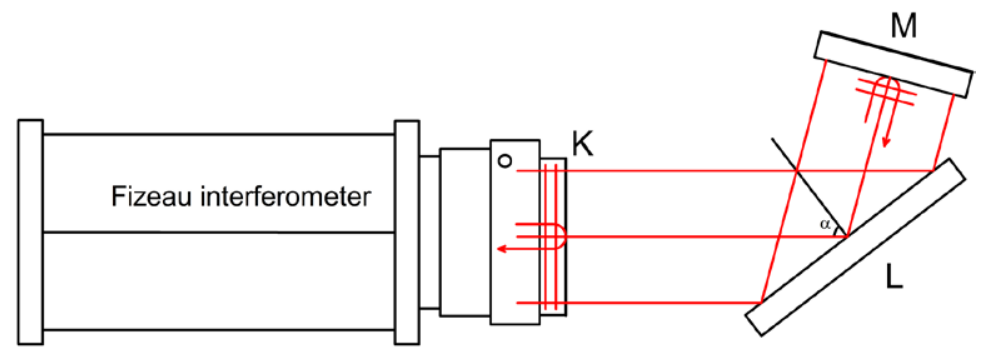

Figure 1. Optical setup proposed for single profile absolute flatness measurement: (a) first measurement, cavity with the two auxiliary mirrors; (b), second measurement, cavity with the test mirror placed between the two auxiliary flats in non-normal incidence setup.

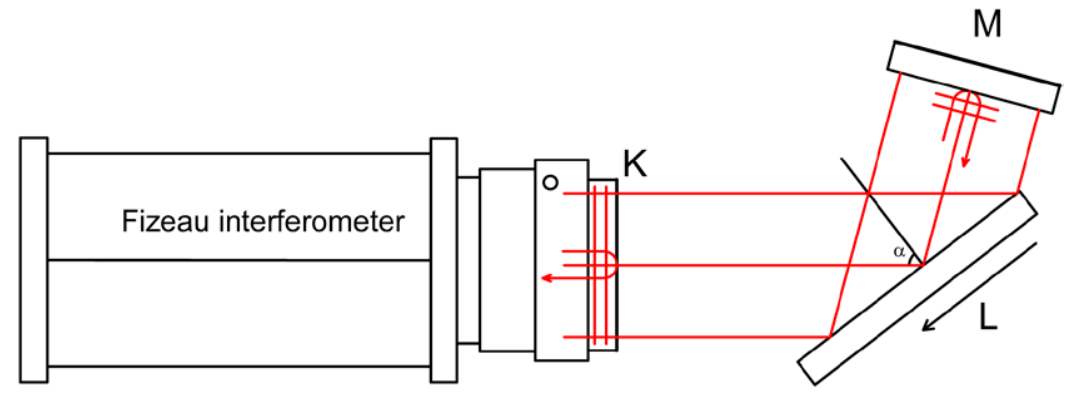

Figure 2. Third measurement, identical to the second one but with a small translation of the mirror $L$ along its length.

One of the reference methods is the three-flat test [7-11]. Three nominally flat surfaces are examined, comparing each other in a particular sequence, introducing also a rotation, for a total of four measurements. The interferograms are then processed with a suitable algorithm to work out the absolute shape of all the three flats. When only the measurement of one of them would be interesting, a particular scheme with only three measurements has been proposed [12]. This method has the advantage of the flats remaining in their mechanical assembly, avoiding any mounting and dismounting from the interferometer and allowing a high stability. Unfortunately, the necessity to do a rotation of one of the flats is remaining. This operation can be a problem for big flats, not only for technical reasons but also because the mechanical constraints against gravity are different and this effect can limit the accuracy of the method.

Methods using only one flat and the test mirror, with two or more measurements carried out after small translations of one optic respect to the other one, have been also proposed [13-16]. In the [13], a FFT method is applied to four derivative maps, obtained from interferometric measurements with corresponding translations of one flat respect to the other one. The reconstruction is called 'quasi-absolute', because the spherical and astigmatic part cannot be retrieved. In the [14-16], the influence of the linear stage is estimated by the measurements according to a simple model. However, a metrological and absolute measurement of the surface is still difficult to be obtained, mainly because of the radius of curvature, that in last generation $\mathrm{x}$-ray optics is particularly important.

We here propose a different scheme that has several advantages. In comparison with the three-flat test [12], it eliminates the need for any rotation in the case that only one 1D profile of the test mirror is of interest. This is often the case with $\mathrm{x}$-ray mirrors, due to the grazing incidence usage and the relatively narrow size of the beam. In comparison with other differential approaches [13-16], it delivers an absolute profile without having to correct the stage contribution but measuring directly the spherical contribution; using a non-normal incidence setup, it is not limited by the Fizeau aperture but it can be used for longer mirrors: in many cases, stitching can be avoided. An example of measurement, with a comparison with another metrology lab, is presented: the two measurements are agreeing on the $0.1 \mathrm{~nm}$ level, a very high precision level when we compare with previous literature [17].

\section{Radius of curvature measurement}

If we repeat the measurements already shown in previous paper [12] but without using the rotation, we have information only on the symmetric part of the profile figure. For clarity, in figure 1 we report the setup we used.

We indicate as $K(x), M(x)$ and $L(x)$ the planarity error of the three surfaces along their 1D profile, taken for example in correspondence of their center, with $x$ axis locally defined on 
Table 1. Ion-beam polished x-ray mirror.

\begin{tabular}{ll}
\hline Substrate material & Silicon $<100>$ \\
Dimensions & $150 \times 40 \times 40 \mathrm{~mm}^{3}$ \\
Clear aperture & $120 \times 20 \mathrm{~mm}^{2}$ \\
Polishing & Classical + ion-beam polishing \\
\hline
\end{tabular}

Table 2. Interferometric system and optics specifications.

\begin{tabular}{|c|c|}
\hline \multicolumn{2}{|l|}{ Fizeau interferometer } \\
\hline Measuring principle & Phase-shift interferometry \\
\hline Aperture & $101.6 \mathrm{~mm}$ \\
\hline Source & $\begin{array}{l}\text { He-Ne laser, at the wavelength } \\
\lambda=632.8 \mathrm{~nm}\end{array}$ \\
\hline Repeatability & $<0.25 \mathrm{~nm}(2 \sigma)$ \\
\hline Resolution & $\begin{array}{l}\lambda / 12000 \text { (high-resolution mode, double } \\
\text { pass) }\end{array}$ \\
\hline Image size & $1200 \times 1200$ pixels \\
\hline Digitization & 10 bits \\
\hline \multicolumn{2}{|l|}{ Flats } \\
\hline $\begin{array}{l}\text { Clear aperture } \\
\text { diameter }\end{array}$ & $101.6 \mathrm{~mm}$ \\
\hline Material & Fused silica \\
\hline Nominal quality & $\lambda / 20$ \\
\hline
\end{tabular}

each surface. From the measurements shown on figure 1, we have the following set of equations:

$$
\begin{gathered}
K L M(x)=K(-x)+\frac{L(x)}{c f}+M(-x), \\
K M(x)=K(-x)+M(x),
\end{gathered}
$$

in which we preferred to put everything explicit without using the formalism of flipping and scaling operators [11]. The $(-x)$ argument indicates a flipping of the coordinate system, while

$$
c f=\frac{1}{2 \cos (\alpha)},
$$

is the correction factor due to the non-normal incidence. In case of $\mathrm{x}$-ray mirrors, they are often longer than the available beam diameter, and to measure the full aperture with the best sensitivity the best angle of incidence is set following the formula $\alpha=\arcsin (D / X)$, with $D$ the flats diameter and $X$ the length of the X-ray mirror. From the set of equations (1) and (2) it is impossible to derive the absolute profile $L(x)$, but if we compute

$$
c f[K L M(x)-K M(x)]=L(x)+c f[M(-x)-M(x)]
$$

and we take only the symmetric part of the result, the contribution of $M(x)$ is canceled. From the result we can then calculate the curvature of $L(x)$ profile with a proper fitting.

Unfortunately, the procedure shown above is not enough to work out the absolute profile of the optics to sub-nm accuracy. The reason is that we have still an unknown contribution from the term $[M(-x)-M(x)]$ that cannot be wiped out. This error is related to the quality of the flat $M(x)$ : depending on its quality, it cannot be better than several $\mathrm{nm}$ rms [9].

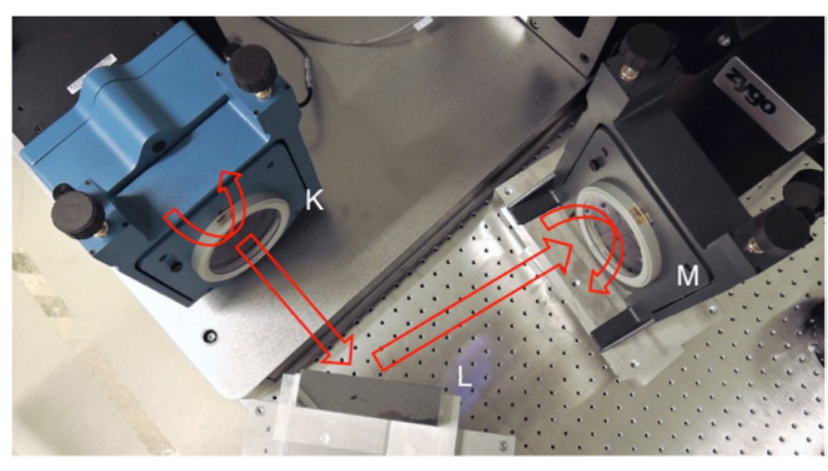

Figure 3. Measuring setup. The red arrows indicate the direction and the reflections of the beam.

\section{Sub-nm accurate profile measurement}

To perform an absolute measurement of the profile with sub-nm accuracy it is needed to add a third measurement, identical to the second one but with a small translation of the mirror under test along its length (figure 2).

If such displacement is $d$, we have:

$$
K L M_{d}(x)=K(-x)+\frac{L(x-d)}{c f}+M(-x) .
$$

Combining equations (1) and (5), we obtain an approximate measurement of the local profile slope:

$$
K L M(x)-K L M_{d}(x)=\frac{L(x-d)-L(x)}{c f} \simeq \frac{d}{c f} L^{\prime}(x),
$$

and we can derive the height profile by integrating the slope $L^{\prime}(x)$.

The final absolute height profile is then worked out combining the information coming from equations (4) and (6), basically to compensate for the unknown spherical contribution introduced by the not perfectly parallel translation. The formula

$$
L(x)=\frac{c f}{d} \int_{0}^{x}\left[K L M(\widehat{x})-K L M_{d}(\widehat{x})\right] \mathrm{d} \widehat{x},
$$

produces some filtering in the spatial frequency domain, as explained in [13]. As a guideline for the setup choice, the first zero of the Modulation Transfer Function is in $\nu_{x}=1 / d$ and a value of $64 \%$ is achieved on $\nu_{x}=1 /(2 d)$. An additional filtering would be introduced also from the pixel square integration, but this is considered negligible if the translation is 15 pixels or more. The filtering effect is comparable to the one occurring on the slope profilometers due to the finite size of the measuring beam, also on the mm scale.

\section{Demonstration of the method}

To test the method, we measured an ion-beam polished silicon mirror using a commercial Fizeau interferometer and two auxiliary flats. The mirror is $150 \mathrm{~mm}$ long while the Fizeau has $100 \mathrm{~mm}$ diameter beam aperture. The auxiliary flats have the same diameter. We report in table 1 the specifications of the mirror and in table 2 the specifications of the Fizeau and the flats. 


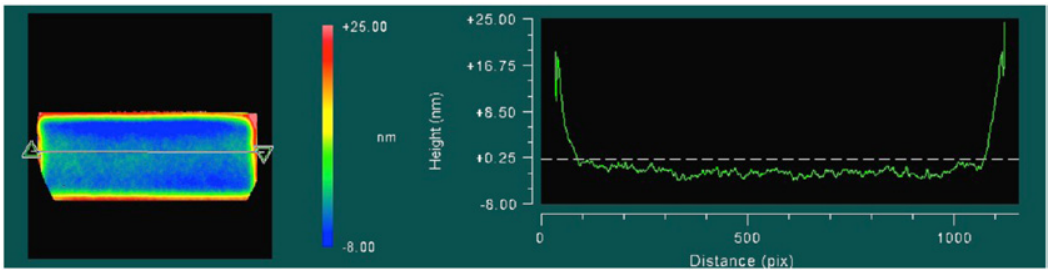

Figure 4. Typical output of the Fizeau interferometer for the non-normal incidence setup. This measurement has been already scaled by the correction factor and the cavity contribution.

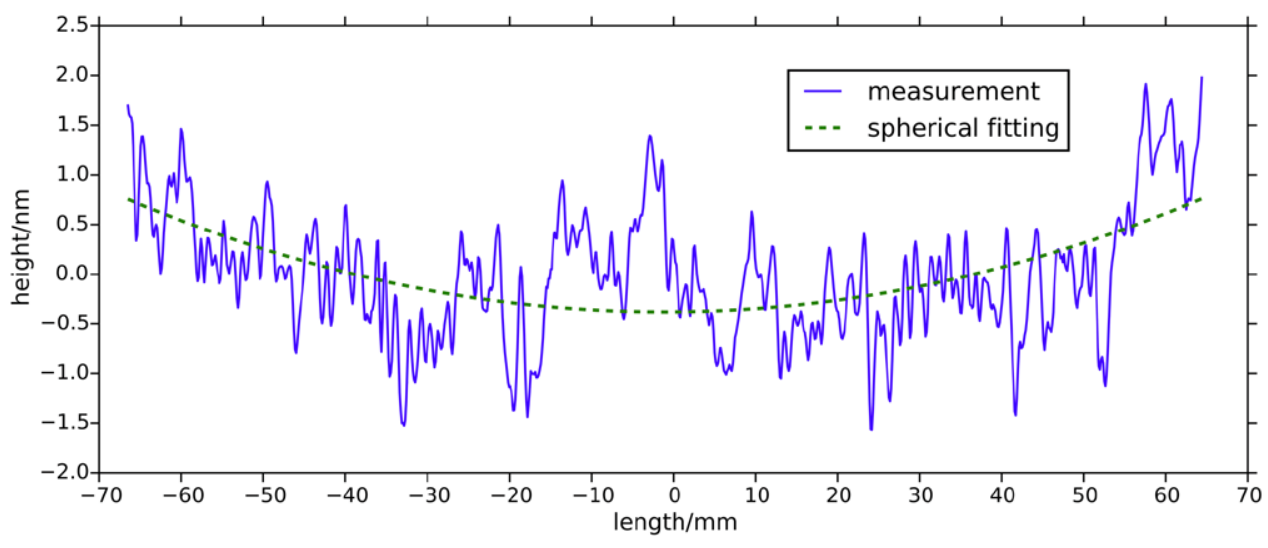

Figure 5. Profile measurement calculated from equation (4) and spherical fitting.

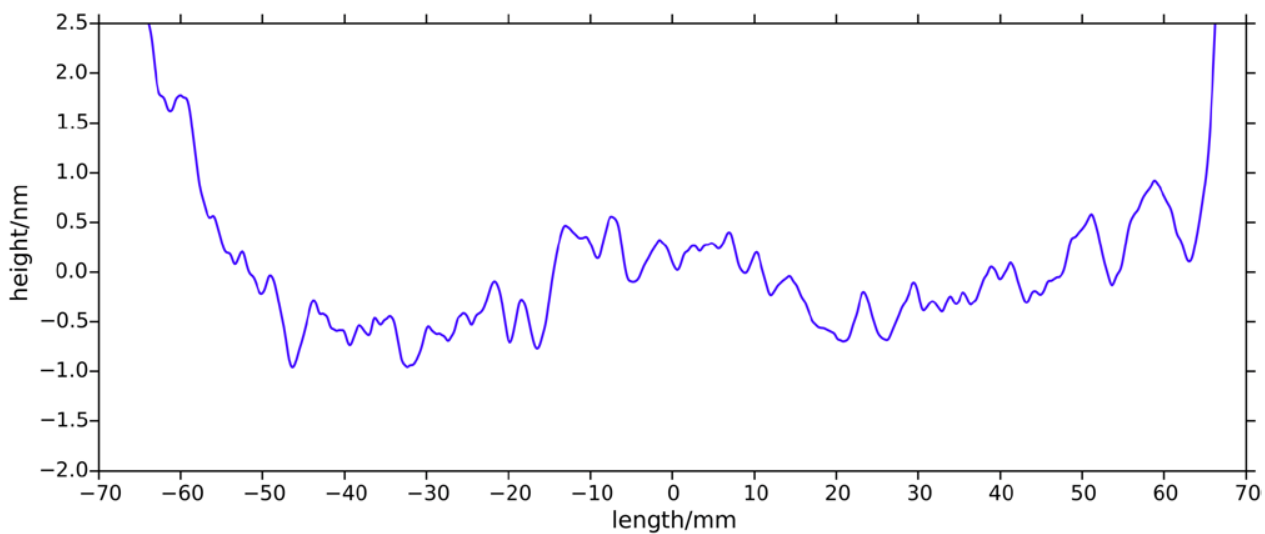

Figure 6. Profile measurement calculated from equation (6) plus the spherical fitting previously calculated.

The measurements sequence is as follow:

(a) The setup with the cavity between the two auxiliary flats is placed and aligned as depicted in figure 1(a). The measurement is performed and the result is stored as 'meas1'.

(b) We insert the silicon mirror and we move the second flat to create the setup in figure 1(b). We align the mirror and the return flat. The measurement is performed and saved as 'meas2'.

(c) The mirror is translated by a small amount, corresponding to about 10 pixels on the interferometer camera and $1.5 \mathrm{~mm}$ in the mirror length axis in this particular example. The two auxiliary flats are not touched nor realigned. The measurement is performed and saved as 'meas3'.

A picture of the setup in non-normal incidence is shown in figure 3.
The typical result is shown in figure 4 as a map. The central profile is then worked out from the map.

The data analysis is then performed in this way:

(a) We calculate the subtraction between 'meas2' and 'meas1'. We correct the resulting map by the correction factor, following equation (4): in this example the angle is about $42^{\circ}$. We extract the central profile from the map and we perform the spherical fitting. The radius of curvature is then calculated: in this example it was $1890 \mathrm{Km}$ (figure 5).

(b) We calculate the subtraction between 'meas3' and 'meas2'. We correct the resulting map by the correction factor and the displacement, to have the slope map as described by the equation (6). The central profile is then extracted and integrated. From the resulting profile, we calculate the spherical contribution and we remove it. Then, we add the sphere calculated in the previous step (figure 6). 


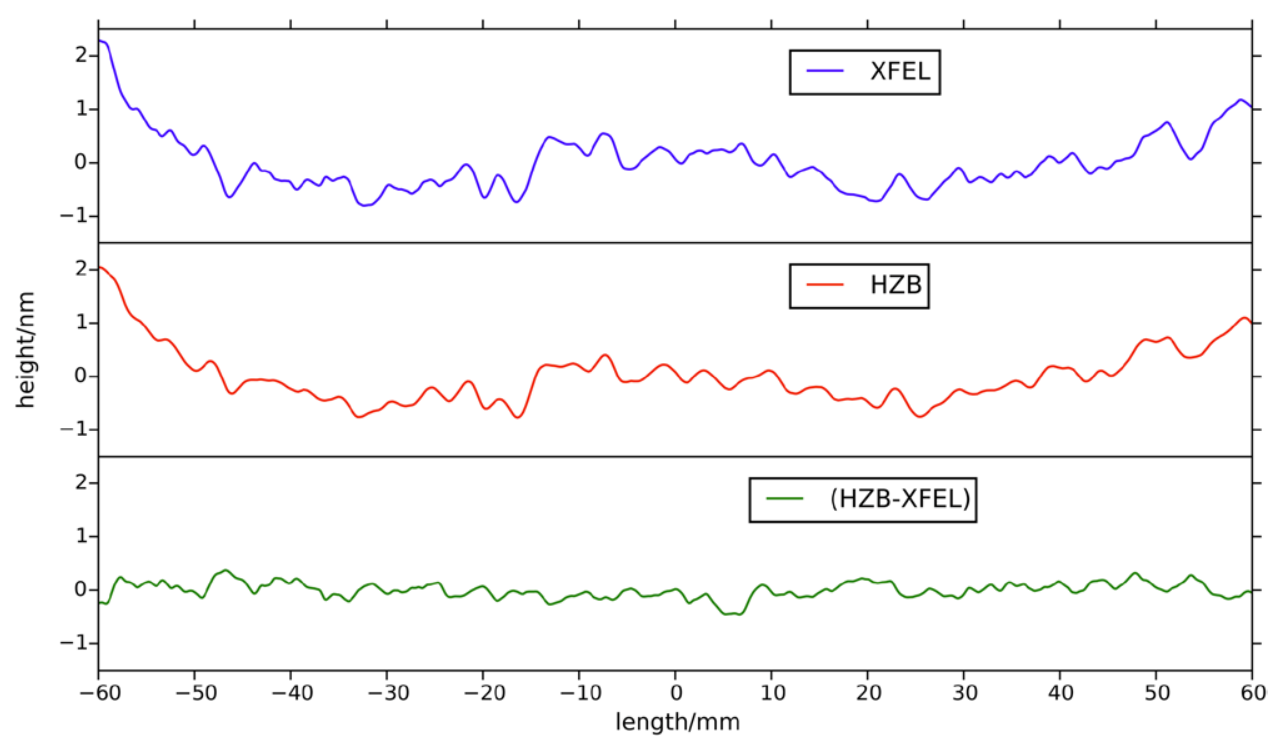

Figure 7. Metrological comparison between our measurement (XFEL) and a calibration measurement (HZB). In the third graph we can see the difference between the two profiles (HZB-XFEL).

The final result is an absolute, highly accurate profile of the mirror surface. If the noise level is low enough, the result has a sub-nm accuracy level and the influence from the auxiliary flats quality is canceled. To have a lower noise result, in every step we averaged 200 interferometer measurements; with our instrument this is relatively easy to do and takes only about $10 \mathrm{~min}$. To judge the quality of the result, we compared this result with the one carried out on another metrology laboratory on the same mirror. The second profile was obtained with a completely different instrument, a sub-nm precision profilometer (NOM) in use in HZB-BESSY laboratory in Berlin, a metrology laboratory for X-ray mirrors with high-resolution slope measurements capability. The result is shown in figure 7, limiting the analysis in the clear aperture of the mirror where the ion beam polishing is most effective. The height difference between the two profiles has a root-mean-square of $0.14 \mathrm{~nm}$ and a Peak-to-Valley of $0.8 \mathrm{~nm}$. As a comparison, the statistics of the profile measured with the NOM is rms $0.51 \mathrm{~nm}$ and P-V $2.8 \mathrm{~nm}$, while the profile measured at XFEL is rms $0.52 \mathrm{~nm}$ and P-V $3.1 \mathrm{~nm}$.

\section{Conclusions}

A method to measure the absolute profile of an x-ray mirror with an oblique incidence setup, using a Fizeau interferometer and two auxiliary flats, has been described. An example of a measurement on a real mirror has been provided, showing a promising indication that sub-nm accuracy (rms) could be reached. We are planning to do an extensive investigation about the influence of stage errors, incidence angle errors, air turbulence, thermal and mechanical drifts in the future. This result places the interferometric method at the same top limit as the current metrology level available with the best highresolution slope measurements. The current limitations of the method are that it is delivering just a single profile and not a 2D-map, even if this profile is reliable and free from systematic errors. Another limitation is the reduced spatial resolution, due to the translation, that can be anyway controlled and optimized in real cases.

In the future, we are planning to apply this method on longer mirrors, up to $1 \mathrm{~m}$. This implies a much longer cavity, so the environment conditions could limit the final accuracy of the method, with the noise and variability of the measurements increasing considerably. The repeatability of the approach should then be assessed. Implementing such a method for long mirrors would be very important for the future x-ray optics projects around the facilities. One of the most interesting issues is to be able to perform a sub-nm measurement using a different method besides long trace profilometry, providing an independent metrological characterization of the mirrors. Due to the limited number of measurements required and to the high speed and effectiveness of the data reduction processing, the method here described could be of value not only for final characterization, but also to assist the manufacture of high quality mirrors by deterministic polishing, which is often optimized for the central profile of the mirror.

\section{Acknowledgments}

We would like to thank F Siewert (HZB, Bessy II, Berlin) for the high quality characterization of the mirror that we used as a comparison.

\section{References}

[1] Weiser M 2009 Ion beam figuring for lithography optics Nucl. Instrum. Methods Phys. Res. B 267 1390-3

[2] Arkwright J, Burke J and Gross M 2008 A deterministic optical figure correction technique that preserves precisionpolished surface quality Opt. Express 16 13901-7

[3] Arnold T, Böhm G, Fechner R, Meister J, Nickel A, Frost F, Hänsel T and Schindler A 2010 Ultra-precision surface finishing by ion beam and plasma jet techniques—status and outlook Nucl. Instrum. Methods A 616 147-56 
[4] Mori Y, Yamauchi K and Endo K 1987 Elastic emission machining Precis. Eng. $9123-8$

[5] Yellowhair J and Burge J H 2007 Analysis of a scanning pentaprism system for measurements of large flat mirrors Appl. Opt. 46 8466-74

[6] Siewert F, Buchheim J, Boutet S, Williams G, Montanez P Krzywinski J and Signorato R 2012 Ultra-precise characterization of LCLS hard x-ray focusing mirrors by high resolution slope measuring deflectometry Opt. Express 20 4525-36

[7] Fritz B S 1984 Absolute calibration of an optical flat Opt. Eng. 23 379-83

[8] Hariharan P 1997 Interferometric testing of optical surfaces: absolute measurements of flatness Opt. Eng. 36 2478-81

[9] Vannoni M and Molesini G 2007 Iterative algorithm for three flat test Opt. Express 15 6809-16

[10] Vannoni M and Molesini G 2008 Three-flat test with plates in horizontal posture Appl. Opt. 47 2133-45

[11] Vannoni M, Sordini A and Molesini G 2012 Calibration of absolute planarity flats: generalized iterative approach Opt. Eng. 51081510
[12] Vannoni M 2014 Absolute flatness measurement using oblique incidence setup and an iterative algorithm. A demonstration on synthetic data Opt. Express 22 3538-46

[13] Morin F and Bouillet S 2007 Absolute interferometric measurement of flatness: application of different methods to test a $600 \mathrm{~mm}$ diameter reference flat Proc. SPIE 6616 66164G

[14] Vidal J, Nicolas J and Campos J 2009 Analysis of the positioning error on lateral shearing surface reconstruction with a Fizeau interferometer Proc. SPIE $739073900 \mathrm{~N}$

[15] Vidal J, Nicolas J and Campos J 2009 Methods to improve the accuracy of the surface reconstruction with a Fizeau interferometer Proc. SPIE 7448744802

[16] Vidal J, Nicolas J and Campos J 2011 New method to improve the accuracy in a sequential lateral shearing interferometer Opt. Eng. 50115601

[17] Artemiev N A, Merthe D J, Cocco D, Kelez N, McCarville T J, Pivovaroff M J, Rich D W, Turner J L, McKinney W R and Yashchuk V V 2012 Cross comparison of surface slope and height optical metrology with a super-polished plane Si mirror Proc. SPIE. $\mathbf{8 5 0 1} 850105$ 